\title{
Enfisema subcutâneo durante exodontia de terceiro molar: relato de caso
}

\author{
Emphysema subcutaneous during third molar extraction: case report \\ El enfisema subcutáneo durante la extracción del tercer molar: reporte de caso \\ Pedro Henrique Silva GOMES-FERREIRA ${ }^{1}$ \\ Danila de OLIVEIRA ${ }^{2}$ \\ Gustavo Antônio Correa MOMESSO ${ }^{1}$ \\ João Paulo BONARDI ${ }^{1}$ \\ Claudio Maltonado PASTORI ${ }^{3}$ \\ Leonardo Perez FAVERANI ${ }^{1}$ \\ ${ }^{I}$ Departamento de Cirurgia e Traumatologia Bucomaxilofacial e Clínica Integrada, Faculdade de Odontologia de Araçatuba, UNESP \\ Universidade Estadual Paulista, Araçatuba-SP, Brasil \\ ${ }^{2}$ Graduanda em Odontologia, Faculdade de Odontologia de Araçatuba, UNESP-Universidade Estadual Paulista, Araçatuba-SP, Brasil \\ ${ }^{3}$ Cirurgião Bucomaxilofacial, Hospital Santa Casa de Misericórdia de Marília, Marília, SP, Brasil
}

\section{Resumo}

A exodontia dos terceiros molares é um procedimento muito realizado no consultório odontológica. Dentre as complicações mais frequentes, o edema, dor, sangramento, infecção e lesão do nervo alveolar ou lingual são citados, porém complicações mais raras, tais como enfisema subcutâneo, são passíveis de ocorrer. Sendo assim, este trabalho tem como objetivo relatar um caso de enfisema subcutâneo causado por caneta de alta rotação durante a exodontia de $3^{\circ}$ molar incluso, o qual foi diagnosticado durante o procedimento, apresentando distensão dos tecidos periorbitário e região média da face esquerda com crepitação a palpação. Esta complicação foi tratada satisfatoriamente com uso de antimicrobiano e anti-inflamatório pósoperatório. Sendo assim, os enfisemas subcutâneos em face são passíveis de ocorrer e quando bem tratado com medicações antimicrobianas e anti-inflamatórias, apresentam remissão espontânea em curto prazo.

Descritores: Enfisema Subcutâneo; Terceiro Molar; Cirurgia Bucal.

\begin{abstract}
The third molar extraction is a widely used in the dental office procedure. Among the most frequent complications, edema, pain, bleeding, infection and alveolar or lingual nerve injury are cited, but rare complications such as subcutaneous emphysema, are likely to occur. Thus, this study aimed to report a case of subcutaneous emphysema caused by high-speed handpiece during the impacted third molar extraction, which was diagnosed during the procedure, showing periorbital tissue and left face middle region distension with crackling palpation. This complication was successfully treated with antimicrobial and anti-inflammatory medications. Therefore, face subcutaneous emphysema is likely to occur and when properly treated with antimicrobial and aintiinflamatory drugs, it has spontaneous remission in the short term.
\end{abstract}

Descriptors: Subcutaneous Emphysema; Molar, Third; Surgery, Oral.

\section{Resumen}

La extracción de los terceros molares es ampliamente utilizada en el procedimiento en el consultorio dental. Entre las complicaciones más frecuentes se citan el edema, dolor, sangrado, infección y lesión del nervio alveolar y lingual. Sin embargo, las complicaciones poco frecuentes, como el enfisema subcutáneo, es probable que ocurran. Por lo tanto, este estudio tiene como objetivo presentar un caso de enfisema subcutáneo causado por la pieza de mano de alta velocidad durante la extracción de tercer molar retenido, que fue diagnosticado durante el procedimiento, con distensión del tejido periorbitario y la región media de la cara izquierda con el crepitar de palpación. Esta complicación se trató con éxito con el uso de drogas postoperatorias antimicrobiana y anti-inflamatoria. Por lo tanto, el enfisema subcutáneo en la cara es probable que ocurran y cuando se trata adecuadamente con fármacos antimicrobianos y anti-inflamatorio tienen remisión espontánea en el corto plazo.

Descriptores: Enfisema Subcutâneo; Dente Serotino; Cirugía Bucal. 


\section{INTRODUÇÃO}

A exodontia dos terceiros molares é o procedimento mais realizado no consultório odontológica no que se diz respeito a especialidade da Cirurgia e Traumatologia Bucomaxilofacial, e mesmo o cirurgião apresentando habilidades e experiência, esse procedimento é passível de complicações ${ }^{1,2}$.

Dentre as complicações mais frequentes $o$ inchaço, dor, sangramento, infecção e lesão do nervo alveolar ou lingual são citados, porem complicações mais raras, tais como enfisema subcutâneo ${ }^{3}$ e até mesmo pneumomedistino ${ }^{4}$, são passiveis de acontecer.

O enfisema subcutâneo é causado por uma injeção de ar forçada no tecido conjuntivo frouxo, abaixo da derme. Instrumento de alta rotação, utilizado nas ostectomias e odontossecções o qual necessita de ar comprimido ${ }^{5}$, pode causar a distensão do tecido por baixo da camada dérmica, o qual permanecerá localmente na região da cirurgia ou pode ser espalhado ao longo do plano do tecido conjuntivo frouxo. Os resultados clínicos são inchaço local e crepitação à palpação ${ }^{6}$.

Sendo assim, este trabalho tem como objetivo relatar um caso de enfisema subcutâneo causado por caneta de alta rotação durante a exodontia de terceiro molar incluso.

\section{CASO CLÍNICO}

Paciente E.P.N.S., do gênero feminino, 31 anos, leucoderma, a qual se queixava de dores na região dos elementos 28 e 38, temporal, mandibular e otalgia. Com finalidade de diagnóstico foi solicitada radiografia ortopantomográfica, observando-se os terceiros molares inclusos, sendo estes a causa mais provável do desconforto e dores relatadas pela paciente (Figuras 1 e 2).

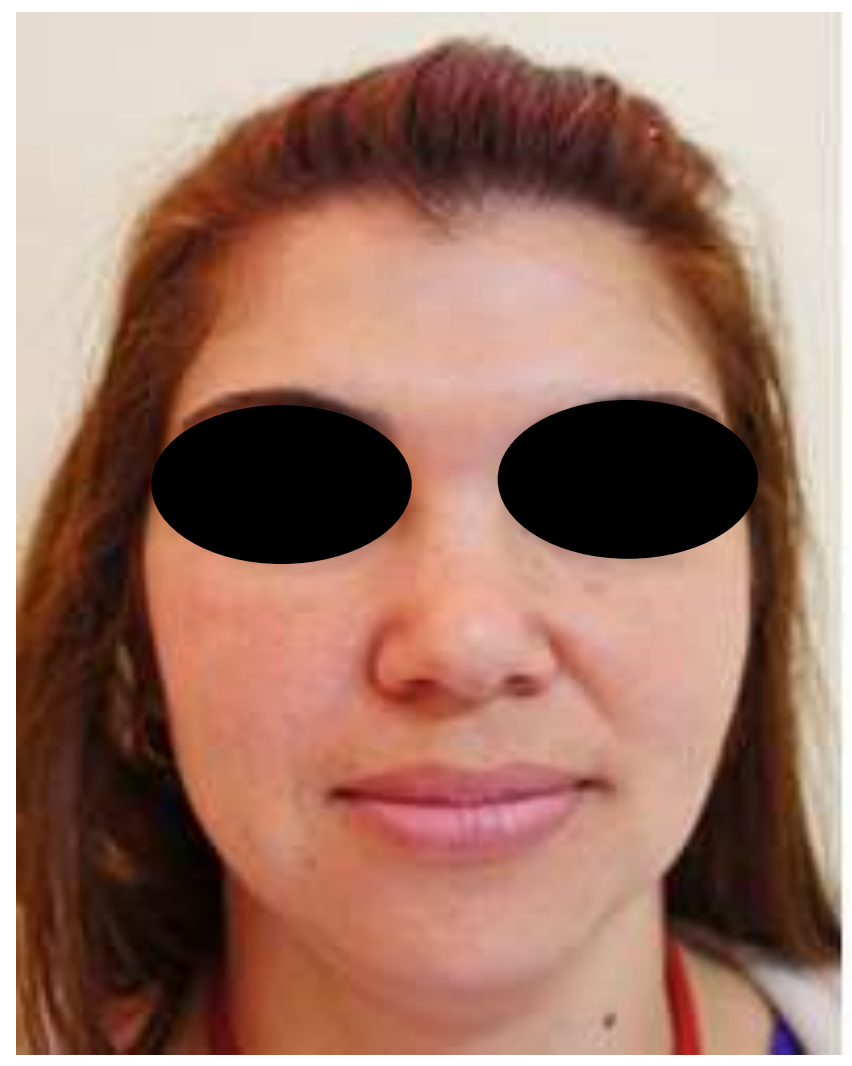

Figura 1. Aspecto clínico

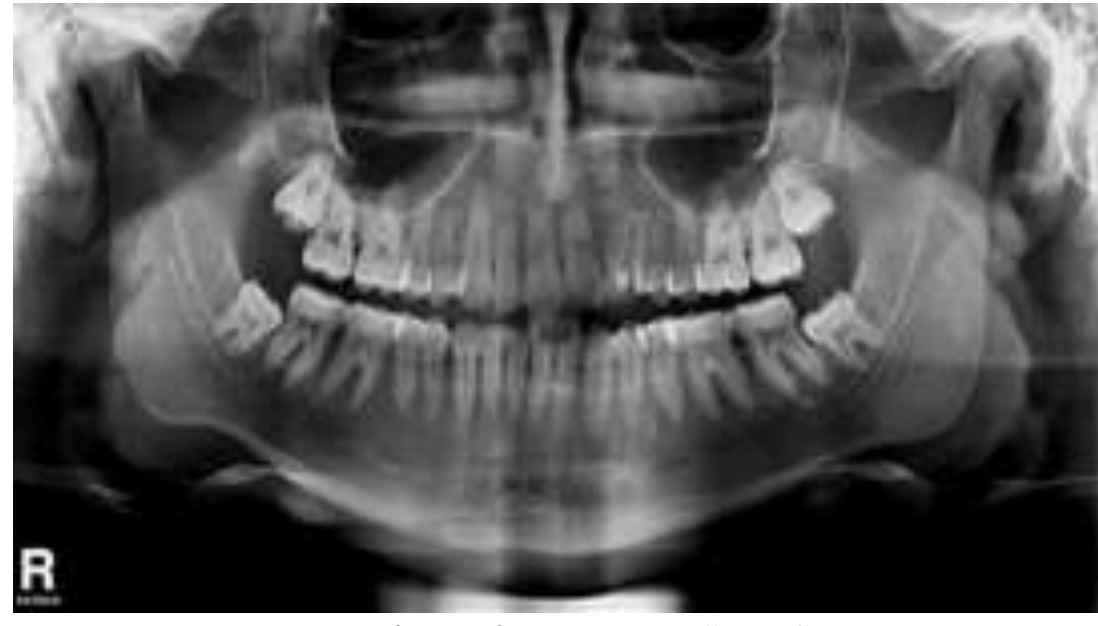

Figura 2. Aspecto radiográfico

Como plano de tratamento, foi estipulado a exodontia do 28 e 38 . Foi instituído como forma de tratamento profilático, a antibioticoterapia previamente a cirurgia e o protocolo medicamentoso preempitvo $1 \mathrm{~h}$ antes do procedimento cirúrgico com corticoide (Dexametasona 8mg).

Primeiramente foi realizada a exodontia do 28 . Durante a remoção do 38, com auxílio de uma peça de alta rotação a paciente relatou alteração da acuidade visual, além de desconforto visual e edema em região infraorbitária esquerda. Ao exame clínico imediato observou-se distensão dos tecidos periorbitário e região média da face esquerda com crepitação a palpação (Figura 3). Aparentemente este enfisema ocorreu pelo ar gerado pela turbina da alta rotação, a qual tem sua saída pela porção posterior da cabeça da caneta, causando assim a distensão dos tecidos na região vestibular da maxila.

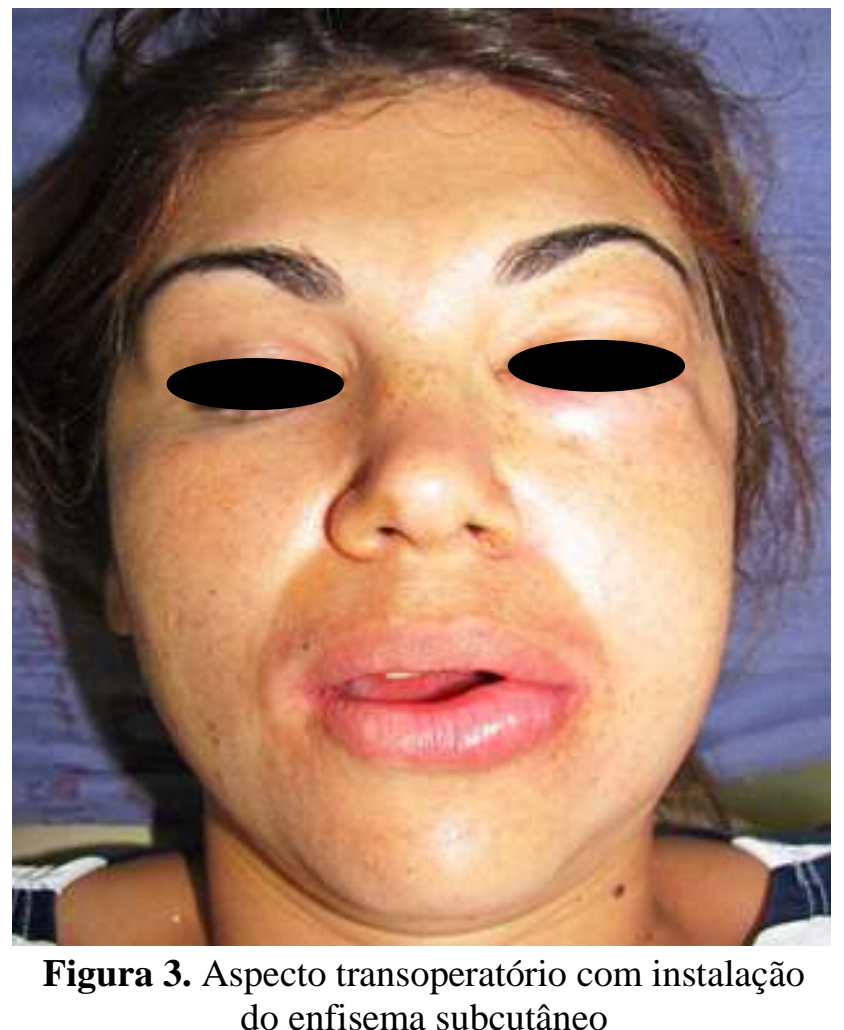

Ao término da cirurgia a paciente permaneceu em observação por duas horas, não sendo constatada evolução do quadro (Figura 4). Foi prescrita medicação antimicrobiana bem como antiinflamatório e analgésico, sendo instituído acompanhamento de possíveis alterações sistêmicas. 


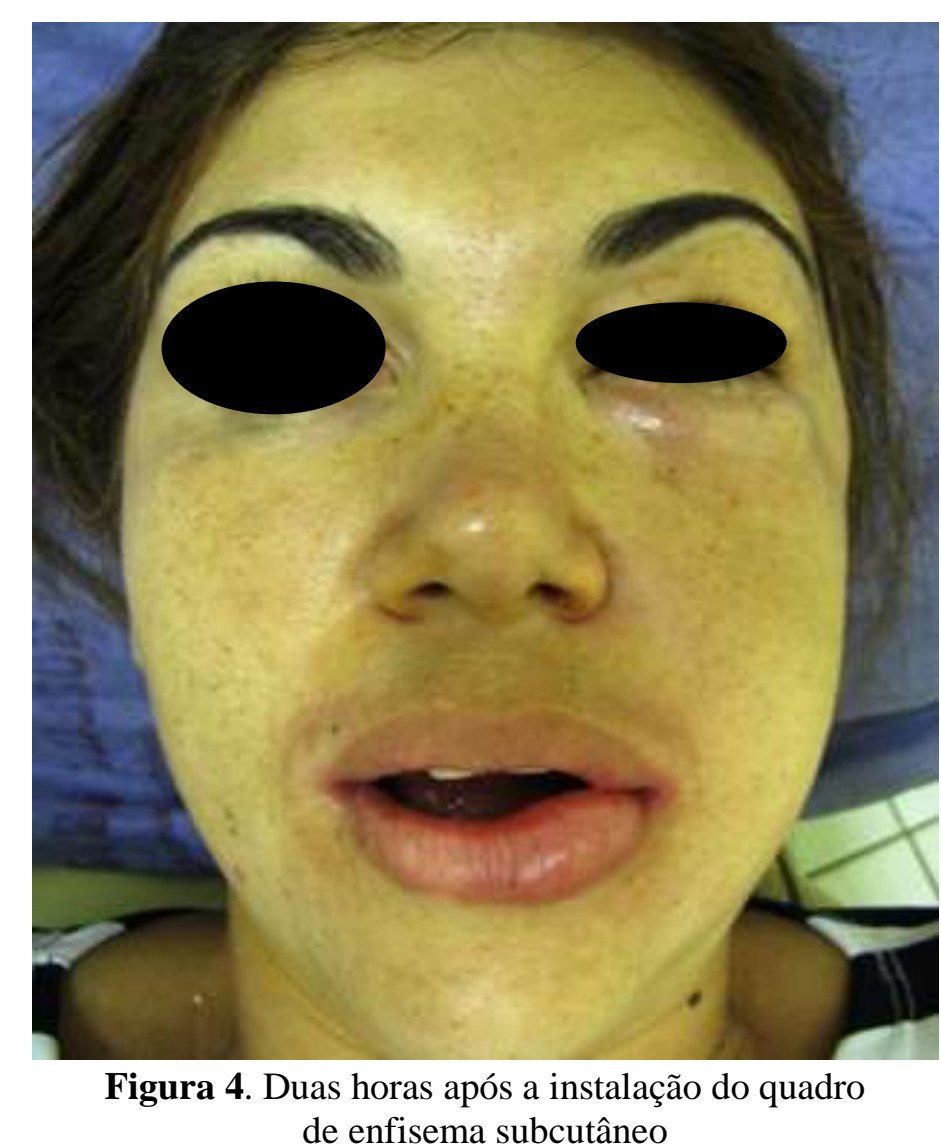

Houve remissão espontânea sem maiores complicações no pós-operatório de 15 dias (Figura 5).

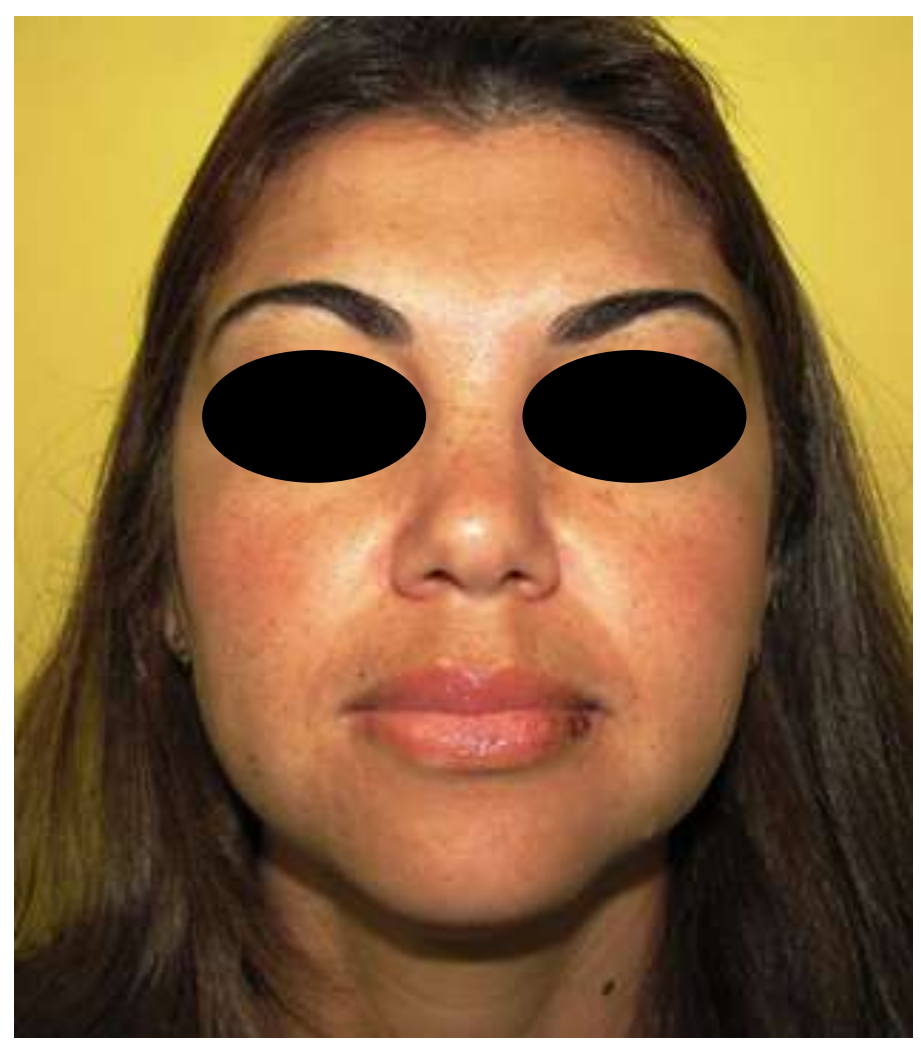

Figura 5. Pós-operatório de 15 dias.

\section{DISCUSSÃO}

Para o correto diagnóstico do enfisema subcutâneo, deve-se avaliar a palpação da área edemaciada, a qual haverá crepitação, ou uma sensação de "velcro", que não é presente nas outras condições ${ }^{7}$. Na maioria dos casos, este sinal é detectado imediatamente, mas há relatos em que podem aparecer posteriormente, tornando $\mathrm{o}$ diagnóstico difícil ${ }^{8}$.

Muitos casos descritos na literatura, trazem como causa desta complicação a remoção dos terceiros molares inferiores, em que o ar da turbina de alguma forma passa pela região inferior através de um caminho livre constituída pelas fibras parcialmente desintegradas do músculo masseter ${ }^{3,9,10}$. Porem o presente caso apresenta como causa aparente a injeção de ar na região de maxila, entre os pontos dados, durante a secção do dente inferior, pois o enfisema chegou a ser palpado até mesmo em região periorbitária.

A intervenção cirúrgica após o diagnóstico de enfisema subcutâneo raramente é tratada de forma cirúrgica. Em alguns casos, podem ser necessárias incisão e drenagem da área afetada com o objetivo de melhorar o desconforto do paciente ${ }^{10}$. Há relato de caso na literatura em que foi realizada a drenagem de tórax em virtude de um pneumotórax bilateral que se manifestou durante a cirurgia de um terceiro mola. ${ }^{11}$ Mesmo sendo necessário estes tratamentos mais invasivos em casos específicos, o tratamento de eleição consiste no acompanhamento e utilização de medicação antimicrobiana e anti-inflamatória associada, como descrito no presente caso.

Mesmo sendo rara as complicações decorrentes do enfisema subcutâneo, podem colocar a vida do paciente em risco ${ }^{12}$. Quando o enfisema atinge espaços pré-traqueal e retrofaríngeos, chegando à região do tórax, pode haver complicações cardiorrespiratórias graves, como o enfisema mediastinial e o pneumotórax ${ }^{10}$. Estas complicações podem gerar, pressão intrapleural, desvio do mediastino, colapso do pulmão entre outros ${ }^{13}$. Mesmo sendo raro o enfisema subcutâneo durante a exodontia de terceiros molares, o cirurgião deve estar sempre atento, pois quando presente, a cirurgia deve ser interrompida para que não haja agravamento piores como os supracitados.

\section{CONCLUSÃO}

De acordo com o exposto, casos de enfisema subcutâneo em face são passíveis de ocorrência. Quando bem tratados com medicação antimicrobiana e anti-inflamatória apresentam remissão espontânea em curto prazo.

\section{REFERÊNCIAS}

1. Kunkel M, Morbach T, Kleis W, Wagner W. Third molar complications requiring hospitalization. Oral Surg Oral Med Oral Pathol Oral Radiol Endod. 2006;102(3):300-6.

2. Jerjes W, Upile T, Nhembe F, Gudka D, Shah P, Abbas $S$, et al.. Experience in third molar surgery: an update. Br Dent J. 2010;209(1):E1.

3. Romeo U, Galanakis A, Lerario F, Daniele GM, Tenore G, Palaia G. Subcutaneous emphysema during third molar surgery: a case report. Braz Dent J. 2011;22(1):83-6.

4. Picard M, Pham Dang N, Mondie JM, Barthelemy I. Cervicothoracic Subcutaneous Emphysema and Pneumomediastinum After Third Molar 
Extraction. J Oral Maxillofac Surg. 2015 Dec;73(12):2286.e1-3

5. Arai I, Aoki T, Yamazaki H, Ota Y, Kaneko A. Pneumomediastinum and subcutaneous emphysema after dental extraction detected incidentally by regular medical checkup: a case report. Oral Surg Oral Med Oral Pathol Oral Radiol Endod. 2009;107(4):e33-e8.

6. McKenzie WS, Rosenberg M. Iatrogenic subcutaneous emphysema of dental and surgical origin: a literature review. J Oral Maxillofac Surg. 2009;67(6):1265-8.

7. Salib RJ, Valentine DLO, Atchtar S. Surgical emphysema following dental treatment. J Laryngol Otol. 1999;133(8):756-8.

8. Gamboa Vidal CA, Vega Pizarro CA, Almeida Arriagada A. Subcutaneous emphysema secondary to dental treatment: case report. Med Oral Patol Oral Cir Bucal. 2007;12(1):76-8.

9. Smatt Y, Browaeys H, Genay A, Raoul G, Ferri J. Iatrogenic pneumomediastinum and facial emphysema after endodontic treatment. Br J Oral Maxillofac Surg. 2004;42(2):160-2.

10. Kung JC, Chuang FH, Hsu KJ, Shih YL, Chen CM, Huang IY. Extensive subcutaneous emphysema after extraction of a mandibular third molar: a case report. Kaohsiung J Med Sci. 2009;25(10):562-6.

11. Sequine J, Irie A, Dotsu H, Inokuchi T. Bilateral pneumothorax with extensive subcutaneous emphysema manifested during third molar surgery. A case report. Int J Oral Maxillofac Surg. 2000; 29(5):355-7.

12. Gulati A, Baldwin A, Intosh IM, Krishnan A. Pneumomediastinum, bilateral pneumothorax, pleural effusion, and surgical emphysema after routine apicectomy caused by vomiting. Br J Oral Maxillofac Surg. 2008;46(2):136-7.

13. Rivas de Andrés JJ, Lópes MFJ, López-Rodó LM, Trullén AP, Lanzas JT. Guideline for the diagnosis and treatment of spontaneous pneumothorax. Arch Bronconeumol. 2008;44(8):437-48.

\section{CONFLITO DE INTERESSES}

Os autores declaram não haver conflitos de interesse.

\section{AUTOR PARA CORRESPONDÊNCIA}

Pedro Henrique Silva Gomes-Ferreira

pedroferreirabmf@gmail.com

Submetido em 29/01/2016 Aceito em 04/02/2016 